\title{
Archiving 3D Cultural Objects with Surface Point-Wise Database Information
}

\author{
N. Tsirliganis, G. Pavlidis, A. Koutsoudis, D. Papadopoulou, \\ A. Tsompanopoulos, K. Stavroglou, Z. Loukou, C. Chamzas* \\ \{tnestor,gpavlid,akoutsou,dpapa,tsompano,kstavrog,rloukou,chamzas\}@ceti.gr \\ Cultural and Educational Technology Institute, http://www.ceti.gr \\ 58 Tsimiski Str., Xanthi 67100, Greece
}

\begin{abstract}
Recent developments in $3 D$ technologies and measurement instrumentation combined with multimedia databases offer today new possibilities for the integrated and complete description of Cultural Heritage objects. A first attempt is made to develop a database for archaeological ceramic and glass artifacts where in addition to the digitized $2 D$ and $3 D$ images, description, typological characteristics and historical information for each artifact will also include point-wise surface data. As a first example, we will include physicochemical data mapped on the surface of the $3 D$ digital image of the object. Thus, the researcher will have the entire information regarding the specific artifact at his disposal. This information will contribute significantly to the comparative study of artifacts, provenance studies, determination of weathering, authentication and detection of forgery, inspection of past restorations etc.
\end{abstract}

\section{Introduction}

The advent of new technologies and their applications revolutionized the way information is stored, archived, retrieved and presented. Their impact on the registration, documentation, presentation and ultimately preservation of Cultural Heritage could be enormous. Systematic recording of the physical and chemical characteristics, typological description and historical information of cultural objects led to the first databases, mainly for research purposes.

Digitization of 2D images of the objects improved the stored information (a picture is always worth a thousand words) and made it more appealing even to the public. Physical and chemical characteristics were still interesting only to a limited number of researchers, but the image combined with historical excerpts in the form of a digital

\footnotetext{
* Corresponding author
}

catalog became a standard in promoting private collections and museums. When catalogs described objects to a greater extend and went deeper into scientific facts, they were used also for educational purposes and typological research $[6,7]$.

Multimedia brought a new era with virtual worlds. The relatively simple catalogs, enriched with video and graphics, transformed to virtual museums, while multimedia databases offered now a multitude of information. Still, even today, this wealth of information remains to a great extend bound to a $2 \mathrm{D}$ world.

However, great advances in 3D technologies offer today new opportunities to record our Cultural Heritage with high precision, in every detail, and present it in an attractive way where the typical "do not touch" caution, has become a bold "please touch and examine".

Moreover, it is not only the new imaging methods that help in the documentation and preservation of Cultural Heritage. Innovations in instrumentation give today more accurate, point-wise measurements of physicochemical characteristics and mechanical properties of objects. Combination of such measurements, 3D imaging and mapping provide a field for the development of new ways to register and present information that can revolutionize once again the documentation of Cultural Heritage. The documentation now will be integrated and complete since we have the ability to describe, digitally store, and retrieve the object not only macroscopically, but also in a point-size fashion that enables the virtual reconstruction of the object in every conceivable detail. The effect that such a reconstruction will have upon scientific research, dissemination of the knowledge and public interest is profound.

A first attempt to this end is done in C.E.T.I. with the collaboration of the Archaeometry Laboratory and the Multimedia Unit for the development of a multimedia database for archaeological ceramics. The database will include detailed 2D and 3D images of archaeological ceramic finds accompanied by morphological descriptions, historical data, and scientific data such as dating 
measurements, mechanical properties and stoichiometric analysis that —where appropriate and possible - will be mapped on the 3D image. Thus, the user-researcher should be able to examine the object from every aspect avoiding at the same time to subject the artifact to any further strain. In addition, the entire object will be stored and be available indefinitely in time and space.

The idea is rather revolutionary since presently the majority of the existing databases concerning cultural \& historical heritage include only 2D images or drawings of objects and are forced to present several images of different visual aspects in order to provide complete representations.

\section{Database}

The design of a cultural database requires the solution of several problems that can be divided into three main categories:

First is the precise definition of the "problem" (theoretical concept), that is

- isolation of the various classes and objects into abstractions and

- definition of the connections or relationships between them.

This is the least technical of the three categories but the most basic one since decisions made in this stage have to be followed through the end.

Second is the implementation, using one or more of the available tools. Common solution here is the use of the relational database model, which works by defining the various classes into "tables", linked to each other according to the relationships of the classes.

Finally there is the user aspect. The target audience must be defined in terms of their demands and knowledge and the user interface must be friendly enough to help them, although (depending again on their demands) it must be rich in terms of available options so the user can ask different questions to the system.

The designer of a cultural database must consider also the possible limitations imposed either from the data involved or from the database/archiving system used. In the present project, the database has to support multimedia content and multilanguage-polytonic text for textual data. Specifically, for the multilanguage-polytonic text requirements the chosen solution was the use of Unicode fonts (although occasional problems are expected since presently, Unicode [8] is not widespread among the users). For enhanced flexibility in database management and data "warehousing" the adopted solution for the multimedia content was to keep them in separate files on disk and include only their filenames with full paths in the database.

When dealing with archaeological objects there are two basic categories of data: cultural and technological. The cultural data comprise external characteristics, such as the type of the object, its dimensions, etc, as well as historical and social information, while the technological data include information related to the chemical, mineralogical and physical properties of the material, archaeological dating information etc.

A summary of the characteristics included in the database is given in table 1. Each of the categories has an open list of sub-categories, with each sub-category being an open list of none, one or more elements.

Table 1. Data types in the database

\begin{tabular}{|c|c|}
\hline Cultural Data & Technological Data \\
\hline $\begin{array}{c}\text { Object type } \\
\text { (lycithos, pithos etc) }\end{array}$ & $\begin{array}{c}\text { Archaeological dating } \\
\text { (measurement method, results) }\end{array}$ \\
\hline $\begin{array}{c}\text { Provenance } \\
\text { (e.g. Corinthian) }\end{array}$ & $\begin{array}{c}\text { Chemical content } \\
\text { (elemental) }\end{array}$ \\
\hline $\begin{array}{c}\text { Historical era } \\
\text { (e.g. Neolithic) }\end{array}$ & $\begin{array}{c}\text { Mineralogical content } \\
\text { (composition) }\end{array}$ \\
\hline $\begin{array}{c}\text { Morphological } \\
\text { characteristics } \\
\text { (shape, size, color, } \\
\text { decoration etc) }\end{array}$ & $\begin{array}{c}\text { Physical properties } \\
\text { (porosity, hardness, } \\
\text { plasticity, strength etc) }\end{array}$ \\
\hline $\begin{array}{c}\text { Present condition } \\
\text { (fragments, whole etc) }\end{array}$ & $\begin{array}{c}\text { Surface traits } \\
\text { (color, pigments, inks, texture etc) }\end{array}$ \\
\hline $\begin{array}{c}\text { Restoration \& } \\
\text { Conservation work }\end{array}$ & $\begin{array}{c}\text { Structure-Microstructure } \\
\text { Excavation data }\end{array}$ \\
\hline $\begin{array}{c}\text { Other characteristics } \\
\text { (e.g. firing temperature, } \\
\text { vessel content etc) }\end{array}$ \\
\hline $\begin{array}{c}\text { (museum, private } \\
\text { collection etc) }\end{array}$ & $\begin{array}{c}\text { Erosion and environmental effects } \\
\text { (patina, cracks etc) }\end{array}$ \\
\hline
\end{tabular}

\section{Presentation of a 3D Cultural Heritage Multimedia Database}

A combination of different technologies was recruited in order to achieve the best possible interaction between the user and the archeological artifacts within a threedimensional environment distributed over a network.

Internet has been acknowledged as one of the most challenging platforms for programming. Java programming language is based on the power of global networks and the idea that the same software should run on many different types of computers and operating systems. A Java application can be easily delivered on the Internet or on any other network. Thus, it is considered as a powerful platform to evolve a 'thin client - thick server' application.

\subsection{D Worlds, Virtual Reality and VRML}

In the late 1960's and 1970's, research on a number of fronts formed the basis of virtual reality as it appears today (e.g. head-mounted displays [4, 5], projection-based VR [2, $3]$ ). In the mid-1980's, the different technologies that enabled the development of virtual reality converged to create the first true VR systems. The term "Virtual Reality" 
was originated at 1989 by Jaron Lanier, the founder of VPL Research, defining it as "a computer generated, interactive, three-dimensional environment in which a person is immersed." Since then, virtual reality has captured the public imagination and lots of work has been done to explore the possibilities of virtual reality in new areas of application such as medicine, chemistry, scientific visualization.

Virtual reality is more than just interacting with $3 \mathrm{D}$ worlds. By offering presence simulation to users as an interface metaphor, it allows operators to perform tasks on remote real worlds, computer generated worlds or any combination of both. The simulated world does not necessarily have to obey natural laws of behaviour. Such a statement makes nearly every area of human activity a candidate for a virtual reality application.

The Virtual Reality Modeling Language (VRML) and Java provide a standardized, portable and platform independent way to render dynamic, interactive 3D scenes across the Internet. Integrating two powerful and portable software languages provides interactive 3D graphics plus complete programming capabilities plus network access $[17,18,19,21]$. The Web is being extended to three spatial dimensions thanks to VRML, a dynamic 3D scene description language that can include embedded behaviors and camera animation. A rich set of graphics primitives provides a common-denominator file format which can be used to describe a wide variety of $3 \mathrm{D}$ scenes and objects. The VRML specification is now an International Standards Organization (ISO) specification [22].

Java adds complete programming capabilities plus network access, making VRML fully functional and portable. This is a powerful new combination, especially as ongoing research shows that VRML plus Java provide extensive support for building large-scale virtual environments [9]

However, there were 2 major limitations in VRML 1.0:

- lack of support for dynamic scene animation, and

- no traditional programming language constructs.

Difficult issues regarding real-time animation in VRML 1.0 included entity behaviors, user-entity interaction and entity coordination. VRML 2.0 development tackled these issues directly, using event-driven ROUTEs to connect 3D nodes and fields to behavior-driven sensors and timing. If Java or JavaScript are supported in a VRML browser, they must conform to the formal interface specified in the specification [22] Annexes B and C, respectively. Major browsers now support both. Using Java is the most powerful way for $3 \mathrm{D}$ scene authors to explore the many possibilities provided by VRML [9].

\subsubsection{VRML and Java interfacing}

The VRML 97 standard does not provide support for the development of shared multi-user worlds. Developers may implement the lacking multi-user and network support in the current standard by means of the Java interfaces without necessitating the definition of non-standard extensions to VRML. Although the current VRML standard does not provide explicit support for the development of multi-user worlds, it does, however, provide the developer with two Java programming interfaces which allow for the implementation of multi-user capabilities: Java via VRML's Script node and External Authoring Interface $[9,10,12,13,14,16,22]$ :

- Java via VRML's Script node is well specified and multiple compliant browsers exist. Sometimes is referred to as the JavaScript Authoring Interface (JSAI)

- External Authoring Interface (EAI). Rather than provide Java connectivity from "inside" the VRML scene via the Script node, the EAI defines a Java or JavaScript interface for external applets which communicate from an "external" HTML web browser [20]. EAI applets can pass messages to and from VRML scenes embedded in an HTML page. The primary benefit of the EAI is the ability for direct communications between the encapsulating HTML browser and the embedded VRML browser. The EAI provides an interface between the VRML world and a Java applet residing on the same page loaded in the Web browser. The EAI now allows for four types of access into the VRML scene:

i. access the functionality of the Browser Interface.

ii. send events to eventIns of nodes in the scene.

iii. read last value sent from eventOuts of nodes.

iv. receive notification when events are sent from eventOuts in the scene.

The EAI thus provides all the functionality of the JSAI although being somewhat more difficult to program.

In the context of multi-user client/server VRML applications, the EAI is the interface of choice since an applet executing at the client can provide both the networking capabilities of Java and access to the internal workings of the VRML world through the EAI [10].

Sun released the Java3D class library for 3D graphics programming [15]. Java3D is an API, providing a programming interface for $3 \mathrm{D}$ that is analogous to the Abstract Window Toolkit (AWT) for 2D graphics. Java3D programs are saved as Java byte codes, not as a modeling format.

All interfaces are well matched, well specified, openly available and portable to most platforms on the Internet. VRML scenes in combination with Java can serve as the building blocks of cyberspace. Building large-scale internetworked worlds now appears possible. Using VRML and Java, practical experience and continued success will move the field of virtual reality past speculative fiction and isolated islands of research onto desktops anywhere, creating the next-generation Web [9]. 


\subsection{Presentation of a Cultural Heritage Multi- media Database using Virtual Reality}

One of the most successful applications of VR is in the representation of historical and cultural heritage. The main reason for this success is that in many cases the represented data do no longer exist or are partially destroyed and cannot be viewed in any other way. In addition, the usual photographic (2D) representation often imposes the requirement to present many pictures of an object (taken from a number of different angles) so as to be able to give viewers a complete description.

In order to implement a system that is able to deliver the virtual reality content of a multimedia database and to use a universal format compatible with most internet browsers, one has no other choice, today, than to adopt Virtual Reality Modeling Language (VRML) [1]. VRML is a well established solution, with ISO approval and the ability to run in many different Internet browsers. VRML appears to be even more applicable as its file format is supported by many state-of-the-art 3D applications in such a degree that importing and exporting from one application to the other is just a few mouse-clicks away. This file format is fully compatible with the software and hardware C.E.T.I. is using for the acquisition of 3D geometry and surface information from the archeological artifacts.

The innovation of the proposed scheme is that there is no other multimedia database of cultural heritage with $3 \mathrm{D}$ data that can be accessed through the internet and also be able to provide with specific object data according to the viewer's point of view. A block diagram of the proposed system is depicted in figure 1 . The block diagram of the work carried out by the system is depicted in figure 2 .

The usage of the system can be summarized as a threestage interaction procedure:

- At a first stage, the user is prompted to use the database search engine in order to locate an object or a family of objects matching desired search criteria. The database generates a report of all matching records and prompts the user to select one from the list.

- At a second stage, the user gets specific information concerning the previously selected object and a 3D representation using VRML language.

- At a third stage, the user interacts with the 3D representation of the object, by turning, zooming, and panning, and by clicking on the surface of the object, while the database reports specific surface information (such as erosion or specific element density and other measurements stored in the database).

More technically, a client implemented within a Java applet is used to forward user's requests to a server application via a socket connection. The server application is responsible for constructing an SQL command, which will be forwarded to the multimedia database server. Once again, the server application will collect the data and forward them back to the applet where they will be presented to the user in a typical $2 \mathrm{D}$ environment of text fields.

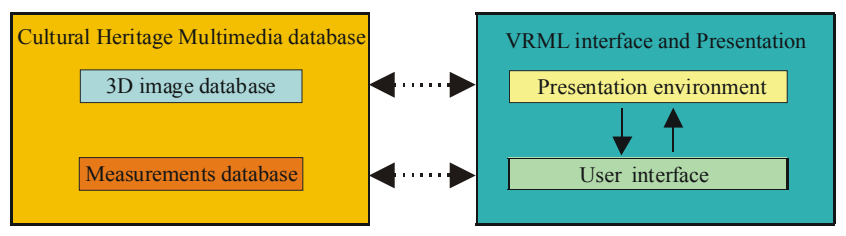

Figure 1. The architecture of the VRML presentation system

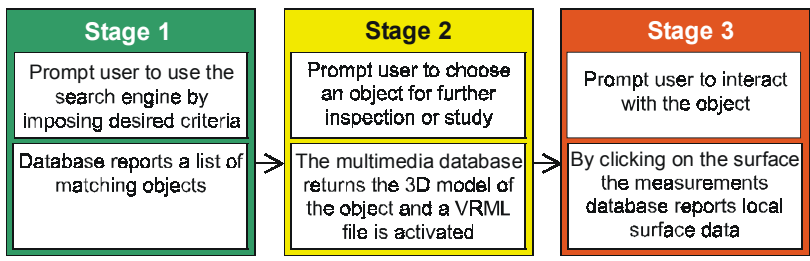

\section{Figure 2. The three-stages system interaction procedure}

The External Authoring Interface (EAI) is the answer to the interactive communication between the VRML world and the Java applet. EAI allows a Java applet to control the contents of the VRML window. Of course, this requires that both VRML window and Java applet be embedded in the same html page. Having those two worlds merged, a powerful platform is achieved. A platform, where high complexity real time 3D graphics coexist with $2 \mathrm{D}$ user interface and parallel supported by database connectivity and distributed application issues.

Summarizing, the technologies used in the proposed implementation are:

- a Java-enabled Web browser (standard, client-side)

- a VRML97 compliant browser plug-in

- a Java applet running in the Web browser (downloaded from the Web server to the client)

- a Java server application (executing on the Web server)

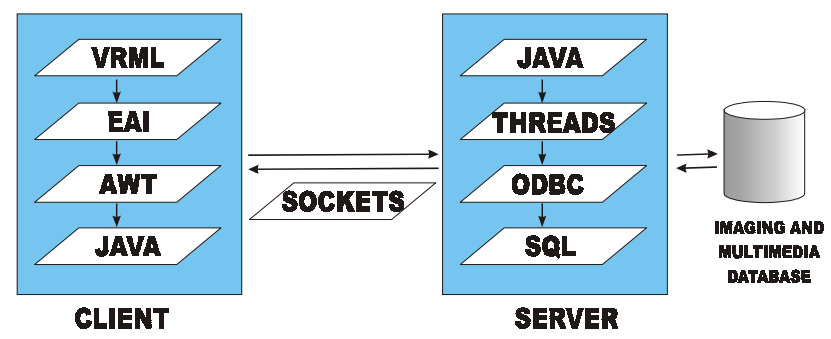

\section{Figure 3. Interaction of different technologies}

Figure 3 is an illustration of the different technologies involved in the present development. The combination of these technologies gives the ability to the end user to run the client on any Operating System platform. Java is highly 
supported by Internet browsers and of course VRML plugins are freely available over the Internet.

The user interacts with the object by a complete freedom of movements (rotation, zooming, panning etc). Selection of a specific area (region of interest) is done by selecting a rectangular region on the $3 \mathrm{D}$ object. With the EAI, the position of the region of interest is transferred to the client applet. The applet forwards the information in the format of a streamed string to the server. The server is now responsible for querying the database. Results are retrieved from the multimedia database, averaged over the selected region and sent back to the client. Apart from the technological data, the server supplies the client with a filename path, which the client uses to download a more detailed 2D image of the region of interest. This image is also presented to the user (figure 4).

In a second phase, the interface will be enhanced with 2D charts of technological data and its functionality will be improved with full control of the VRML world within the applet (preselected viewpoints, preselected-highlighted regions-of-interest, enhanced navigational interface etc). Furthermore, issues related with $3 \mathrm{D}$ data and image compression as well as network security issues currently under investigation will be embodied. Considered technologies include JPEG2000, MPEG4 3D, and future VRML revisions (2002). They are expected to lead in lower

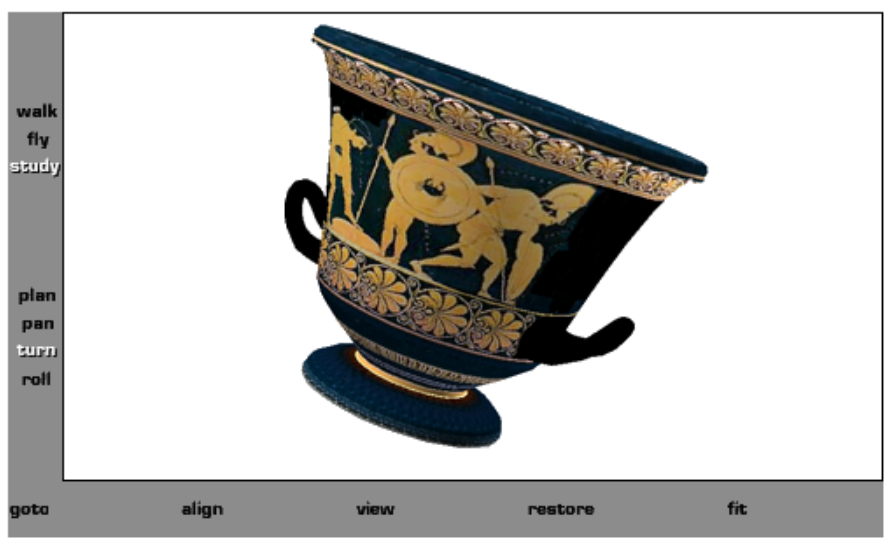

Welcome to 3D ArCAD of CETI

Select region on the surface of the object

Retrieve data

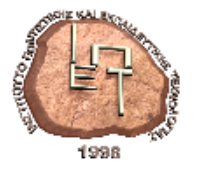

Query SQL
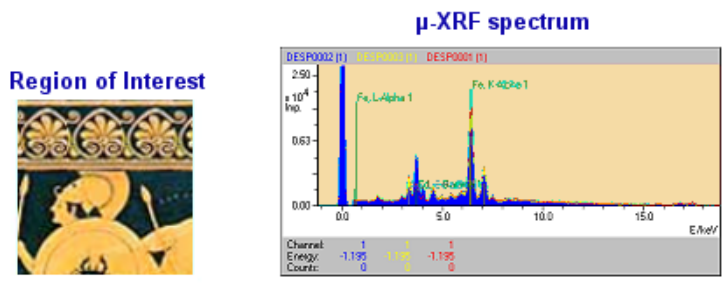

volumes of data transmission and higher speeds of interaction, which will address the most important network issues related with information retrieval in similar systems.

The point-wise surface data are acquired by a micro XRay Fluorescence system ( $\mu$-XRF), which is able to provide with point-wise stoichiometric analysis and elemental content data (figure 5).

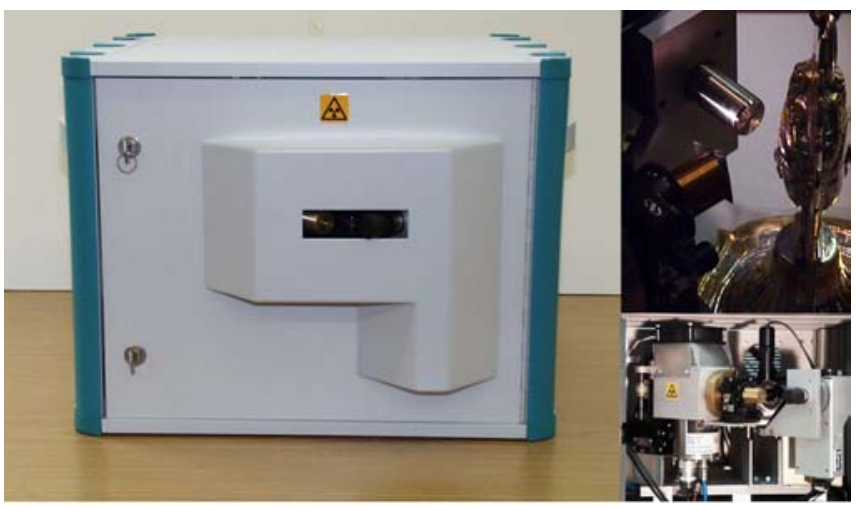

Figure 5 . The $\mu$-XRF point-wise surface data acquisition system

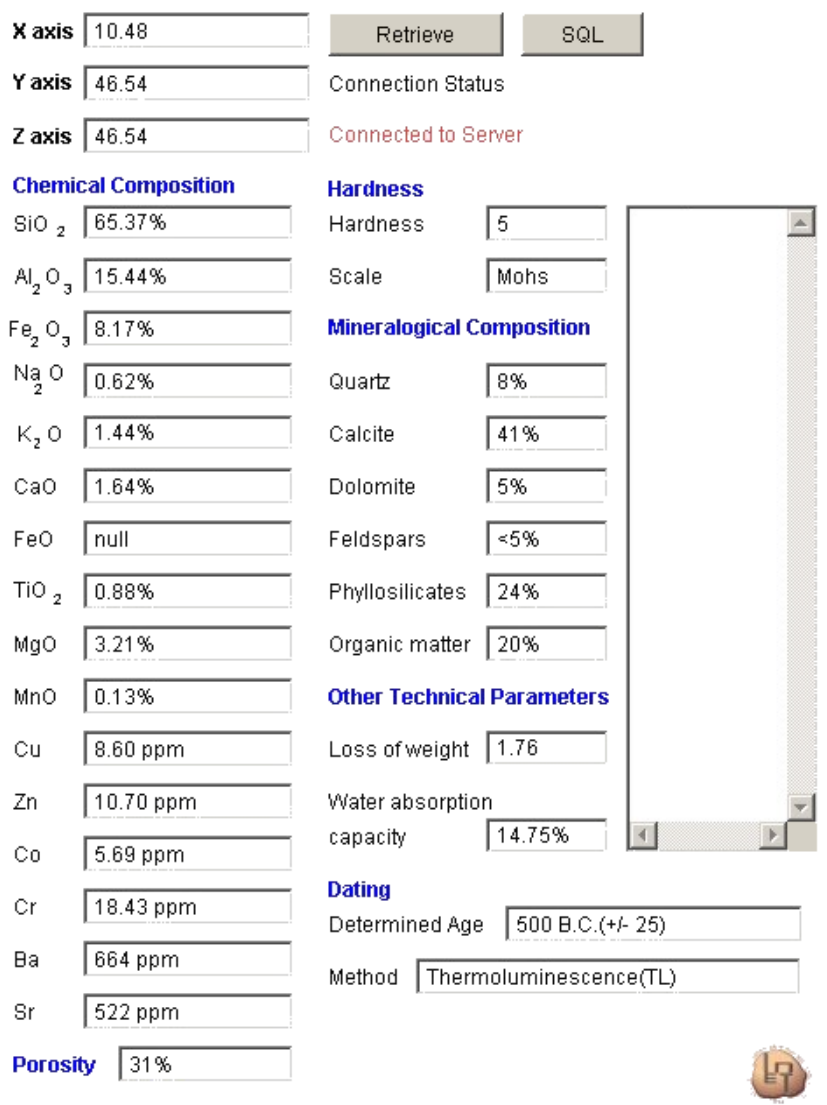




\section{Conclusions}

A multimedia database with real or realistic and interactive 3D representations of objects is under development for archaeological ceramic artifacts. The database contains historical, morphological and analytical data for the recorded artifacts together with their 3D digital images. The researcher/user has access to the entire information related to an artifact and can interact with its $3 \mathrm{D}$ representation by turning, zooming, and panning. In addition, the interested user can retrieve specific local surface data (elemental composition, pigment etc) by clicking on the surface of the object, thus taking advantage of the recent developments in measurement instrumentation to provide an integrated and complete documentation of cultural objects. The database makes use of the "thin clientthick server" programming approach, implemented on a Java platform. External Authoring Interface enables communication with the VRML standard in the client side.

\section{References}

[1] Gobbetti, E. and R. Scateni, Virtual reality: Past, Present and Future.

[2] Krueger, M. W. Responsive environments. In NCC Proceedings (1977), pp. 375-385.

[3] Krueger, M. W. Artificial Reality. Addison-Wesley, 1983.

[4] Sutherland, I. E. The ultimate display. In Proceedings of IFIPS Congress (New York City, NY, May 1965), vol. 2, pp. 506-508.

[5] Sutherland, I. E. A head-mounted three-dimensional display. In Proc. the Fall Joint Computer Conference (1968), pp. 757-764.

[6] Sample databases with typological and historical data: http://www.beazley.ox.ac.uk/test/vases http://www.perseus.tufts.edu/cgi-bin/ http://www.officenet.co.jp/ yoji/vase http://www.art.uiuc.edu/oldkam/GreekKam http://www.lists.bilkent.edu.tr/documentation/pottery/handboo $\mathrm{k} /$ potteryform.html

[7] Sample databases including chemical/geological data: http://Srdweb2.dl.ac.uk:1080/FMRes http://art.sdsu.edu:591/materialbasesearch.html

[8] The Unicode Consortium, The Unicode Standard, Version 3.0, Addison Wesley, 2000 (http://www.unicode.org)
[9] Brutzman, D., The Virtual Reality Modeling Language and Java, Communications of the ACM, vol. 41 no. 6, June 1998, pp. 57-64.

http://www.web3D.org/WorkingGroups/vrtp/docs/vrmljava.pdf

[10] Ferscha, A. and Johnson, J., Distributed Interaction in Virtual Spaces Department of Applied Computer Science, University of Vienna, ferscha@ani.univie.ac.at

[11] http://www.cosmosoftware.com

[12] Ames, Andrea L., Nadeau, David R. and Moreland, John L., VRML 2.0 Sourcebook, second edition, John Wiley \& Sons, New York, 1997.

[13] Carey, Rikk and Bell, Gavin, Annotated VRML 2.0 Reference Manual, Addison-Wesley, Reading Massachusetts, 1997.

[14] Couch, Justin, VermelGen, software distribution, Virtual Light Company, December 1997.

[15] Deering, Michael and Sowizral, Henry, Java3D Specification, Version 1.0, Sun Microsystems Corporation, Palo Alto, California, August 11997.

[16] Hartman, Jed and Wernecke, Josie, VRML 2.0 Handbook, Addison-Wesley, Reading Massachusetts, 1996.

[17] Harold, Elliotte Rusty, Java Network Programming, O'Reilly and Associates, Sebastopol California, 1997. Available software at ftp://ftp.ora.com/published/oreilly/java/java.netprog

[18] Hughes, Merlin, Conrad, Shoffner, Michael and Winslow, Maria, Java Network Programming, Manning Publications, Greenwich England, 1997.

[19] Lea, Rodger, Matsuda, Kouichi and Miyashita, Ken, Java for 3D and VRML Worlds, New Riders Publishing, Indianapolis Indiana, 1996.

[20] Marrin, Chris, External Authoring Interface (EAI) Proposal, Silicon Graphics Inc., Mountain View California, 1997.

[21] Roehl, Bernie, Couch, Justin, Reed-Ballreich, Cindy, Rohaly, Tim and Brown, Geoff, Late Night VRML 2.0 with Java, ZiffDavis Press, MacMillan Publishing, Emeryville California, 1997.

[22] VRML 97, International Specification ISO/IEC IS 14772-1, December 1997, available via www.vrml.org 